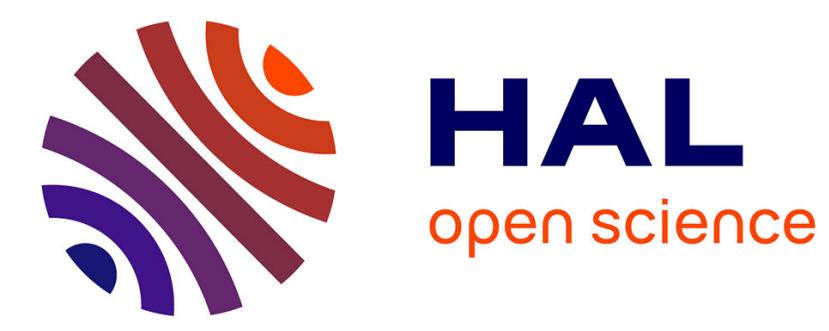

\title{
Cross-helicity in rotating homogeneous shear-stratified turbulence
}

Alexandre Pieri, Fabien S. Godeferd, Claude Cambon, Bérengère Dubrulle, S. Thalabard

\section{> To cite this version:}

Alexandre Pieri, Fabien S. Godeferd, Claude Cambon, Bérengère Dubrulle, S. Thalabard. Crosshelicity in rotating homogeneous shear-stratified turbulence. Physical Review Letters, 2014, 112 (11), pp.114501. 10.1103/PhysRevLett.112.114501 . hal-01296833

\section{HAL Id: hal-01296833 \\ https://hal.science/hal-01296833}

Submitted on 11 Apr 2016

HAL is a multi-disciplinary open access archive for the deposit and dissemination of scientific research documents, whether they are published or not. The documents may come from teaching and research institutions in France or abroad, or from public or private research centers.
L'archive ouverte pluridisciplinaire HAL, est destinée au dépôt et à la diffusion de documents scientifiques de niveau recherche, publiés ou non, émanant des établissements d'enseignement et de recherche français ou étrangers, des laboratoires publics ou privés. 


\title{
Cross-helicity in Rotating Homogeneous Shear-Stratified Turbulence
}

\author{
A. B. Pieri* \\ Institute of Atmospheric Sciences and Climate (ISAC), Corso Fiume 4, I-10133 Torino, Italy \\ F. S. Godeferd and C. Cambon \\ Laboratoire de Mécanique des Fluides et d'Acoustique, École centrale de Lyon, 36 Avenue Guy de Collongue, \\ 69134 Écully cedex, France
}

B. Dubrulle and S. Thalabard

Laboratoire SPHYNX, CEA/IRAMIS/SPEC, CNRS URA 2464, F-91191 Gif-sur-Yvette, France

(Received 17 September 2013; published 18 March 2014)

\begin{abstract}
We consider homogeneous shear-stratified turbulence in a rotating frame, that exhibits complex nonlinear dynamics. Since the analysis of relative orientation between coupled fluctuating fields helps us to understand turbulence dynamics, we focus on the alignment properties of both the velocity and gravity fields with the potential vorticity gradient. With the help of statistical mechanics, we define a vector field which plays a role in the analogous so-called cross-helicity in magnetohydrodynamics. High-resolution direct numerical simulations of developed homogeneous baroclinic turbulence are performed, and a detailed analysis of probability density functions for cross-helicity is provided. A net preference for positive cross-helicity is shown to be related to a new alignment mechanism. We argue that the analysis of cross-helicity is crucial for understanding the dynamics of buoyancy driven flows.
\end{abstract}

DOI: 10.1103/PhysRevLett.112.114501

PACS numbers: 47.32.-y, 05.20.Jj, 47.27.-i

Introduction.-A central feature of geophysical fluid dynamics is due to the so-called baroclinic turbulence, i.e., turbulence under the conjugated action of shear and stratification. This occurs when a global meridional temperature gradient due to differential heating is generated in the atmosphere. Coupled to Earth's rotation, large scale atmospheric currents-thermal winds better known as "jet streams"-are produced at heights about $10 \mathrm{~km}$. At such altitudes, the vertical buoyancy gradient is approximately constant [1] giving tilted isobuoyancy surfaces, i.e., baroclinicity. Under such conditions, available potential energy from the mean meridional density gradient can be converted into turbulent kinetic energy due to horizontal thermal exchanges [2], providing a natural turbulence forcing through such symmetric or baroclinic instabilities. Recent developments in this field can be found in [3-5]. Despite improved understanding of the instability mechanisms, we have not yet reached a satisfactory picture of baroclinic turbulence dynamics [6]. This severely impedes the improvement of large scale atmospheric circulation models.

In the simpler case of homogenous isotropic turbulence, a crucial role seems to be played by the interplay between velocity and vorticity, a phenomenon quantified by the helicity introduced by Moffatt [7] on topological ground. This invariant of inviscid equations of motion is of crucial importance to determine the cascade direction [8] and the so-called depletion of nonlinearity that is observed at large Reynolds number [9-12]. Its counterpart in magnetohydrodynamics (MHD) involves correlations between velocity and magnetic field and is called "cross-helicity." It has recently been used to explain depletion of nonlinear interaction of Alfvén modes [13]. In geophysical turbulence, no equivalent of helicity or cross-helicity was known until a recent work by Gibbon et al. [14]. The authors defined an analog to the magnetic field for buoyancy driven flows as the cross product between the potential vorticity gradient and the buoyancy gradient. The resulting $\mathcal{B}$ vector is solenoidal and is shown to obey a stretching equation similar to the vorticity equation. Cross-helicity must then be understood as representative of the alignment between the fluctuating velocity field $\boldsymbol{u}$ and $\mathcal{B}$. At variance with the MHD case, where only Alfvén waves are present, the shearstratified case includes wave and vortex mode perturbations, coupled in various ways depending on the parameters and on the symmetry properties of the modes $[4,15,16]$.

In the present Letter, we explore further this analogy and study the influence of the cross-helicity on the stationary state of baroclinic turbulence. We find that the final state is in fact governed by a double alignment, one between $\mathcal{B}$ and the gravity, and one between $\boldsymbol{u}$ and $\mathcal{B}$, thereby maximizing the cross-helicity.

Equations of motion.-In the following, background fields are denoted with an overbar, while space-averaged fields are denoted with angular brackets \langle\rangle . We consider a homogeneous rotating fluid in the $f$ plane with additional vertical stratification at constant Brunt-Väisälä frequency $N_{v}^{2}>0$. The Coriolis frequency is twice the rotation rate $f=2 \Omega$. A mean zonal or streamwise shear $S=\partial_{3} \bar{u}_{1}$ is added in background. Due to thermal wind adjustment [17], 


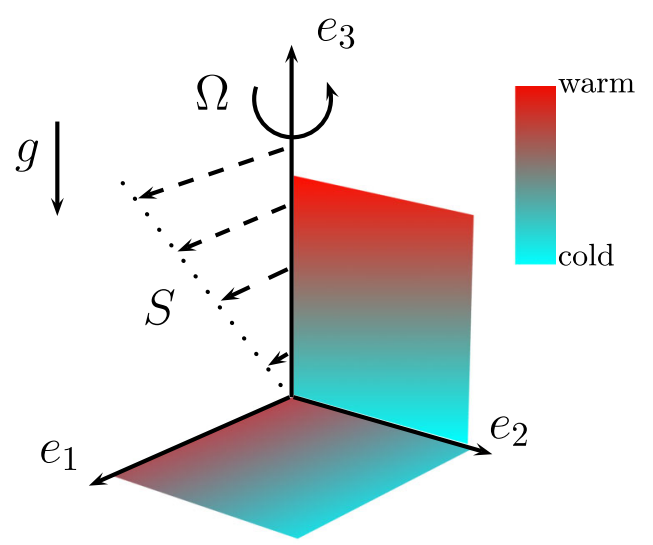

FIG. 1 (color online). Sketch of the mean flow. $S=\partial_{x_{3}} \bar{u}_{1}$ is the shear intensity of the zonal wind. The axis of rotation is chosen parallel to the mean velocity gradient, $\Omega=\Omega \boldsymbol{e}_{3}$. Due to thermal wind adjustment a meridional or spanwise (along $\boldsymbol{e}_{2}$ ) density gradient is enforced.

a meridional or spanwise buoyancy gradient $S f=-\partial_{2} \bar{b}$ is produced. We define the meridional Brunt-Väisälä frequency as $N_{h}^{2}=S f . f, S, N_{v}$, and $N_{h}$ therefore define the background mean state, which remains unchanged throughout the flow evolution. A sketch of this mean flow is presented in Fig. 1. While buoyancy increases with $x_{3}$ (stable stratification), it decreases with $x_{2}$, i.e., going northward.

The fluctuating fields, velocity $\boldsymbol{u}$ and buoyancy $b$, obey the set of incompressible Boussinesq equations,

$$
\begin{aligned}
& \frac{D \boldsymbol{u}}{D t}=-\nabla p-2 \tilde{\Omega} \times \boldsymbol{u}+\nu \nabla^{2} \boldsymbol{u}+\left(b-S u_{1}\right) \boldsymbol{e}_{3}, \\
& \frac{D b}{D t}=-\boldsymbol{u} \cdot \nabla \bar{b}+\chi \nabla^{2} b, \quad \nabla \cdot \boldsymbol{u}=0,
\end{aligned}
$$

where $\tilde{\Omega}=\Omega+\frac{1}{2} \nabla \times \overline{\boldsymbol{u}}$ and $D(.) / D t=\partial_{t}()+.(\overline{\boldsymbol{u}}+\boldsymbol{u})$. $\nabla($.$) .$

Inviscid invariants and statistical mechanics.-Insights about the self-organization of the flows can be obtained by setting $\chi=\nu=0$ in (1), hereby considering inviscid dynamics. From the Boussinesq equations (1) in the inviscid limit, it is straightforward to infer Ertel's theorem $D \Pi / D t=0$, where the potential vorticity $\Pi$ is given by

$$
\Pi=\boldsymbol{\omega}^{(a)} \cdot \nabla(\bar{b}+b),
$$

with $\boldsymbol{\omega}^{(a)}=\nabla \times \boldsymbol{u}+2 \tilde{\Omega}$ the total vorticity. This is the analog of the conservation of vertical vorticity in twodimensional turbulence. From that, one can deduce the conservation of any Casimir of $\Pi, \partial_{t} C_{n}=0$ with $C_{n}$ defined as

$$
C_{n}(\Pi)=\left\langle\Pi^{n}\right\rangle \text {. }
$$

The mechanical energy $\mathcal{E}=\left\langle u^{2} / 2+b^{2} /\left(2 N_{v}^{2}\right)\right\rangle$ for the perturbation is not conserved by the dynamics-even in the inviscid limit-because of the unperturbed base flow [5]. Nevertheless, if the two conservation laws applied together, for the Casimir and energy, they would impose strong constraints on the possible energy transfers and cascade [18] or on the stationary states. For instance, considering only robust quadratic invariants as in twodimensional turbulence [19], one may introduce a free energy $\mathcal{F}$ as the linear combination of the mechanical energy $\mathcal{E}$ (kinetic + potential) and the second order Casimir $C_{2}$,

$$
\mathcal{F}(\boldsymbol{u}, b)=\left\langle\frac{u^{2}}{2}+\frac{b^{2}}{2 N_{v}^{2}}+\mu \frac{\Pi^{2}}{2}\right\rangle,
$$

where $\mu$ is a free parameter. This free energy is a conserved quantity of the inviscid dynamics. Moreover, it can be shown that

$$
\left\langle\Pi^{2}\right\rangle=\langle\boldsymbol{u} \cdot \mathcal{B}\rangle+\langle 2 \tilde{\Omega} \cdot \mathcal{A}\rangle,
$$

where $\mathcal{B}=\nabla \times \mathcal{A}$ is given as

$$
\mathcal{B}=\nabla \Pi \times \nabla(b+\bar{b}) .
$$

The stable steady states are then obtained as minima of the free energy function and obey, for $\delta \mathcal{F}=0$,

$$
\begin{aligned}
& \boldsymbol{u}+\mu \nabla \Pi \times \nabla(b+\bar{b})=0, \\
& \frac{b}{N_{v}^{2}}-\boldsymbol{\omega}^{(a)} \cdot \nabla \Pi+\Pi \frac{\partial \Pi}{\partial b}=0 .
\end{aligned}
$$

The first equation means that $\boldsymbol{u} \times \mathcal{B}=0$. The free energy function is then minimized when the velocity vector is colinear with the vector $\mathcal{B}$. This is the analog of the alignment between the velocity and the magnetic field observed in MHD turbulence and is rooted into a deeper analogy between stratified and MHD turbulence, noticed by Gibbon et al. [14]. In the $f$-plane approximation, there is no mean potential vorticity gradient in the flow so that the $\mathcal{B}$ vector corresponding to the background flow reduces to zero. The $\mathcal{B}$ vector of the total flow is induced only by the presence of turbulent fluctuations.

Analogy with MHD.--Indeed, starting from the definition of $\mathcal{B}$ given in (6), one gets from Eq. (1) in the inviscid limit the stretching equation,

$$
\frac{D \mathcal{B}}{D t}=\mathcal{B} \cdot \nabla(\overline{\boldsymbol{u}}+\boldsymbol{u})
$$

Moreover, $\mathcal{B}$ is divergence free and can be written $\mathcal{B}=\nabla \times \mathcal{A}$, where $\mathcal{A}=\Pi \nabla(b+\bar{b})$. The vector $\mathcal{B}$ is then an analogue of a magnetic field, while $\mathcal{A}$ is the analog of a magnetic potential. Moreover, the equality (5) means that the second-order Casimir is the analog of the cross-helicity, which has been recently shown by [13] to play an essential 
role in MHD turbulence by inhibiting the nonlinear energy cascade when the magnetic and velocity fields are aligned or antialigned. In the following, we therefore exploit this analogy to check whether a similar property holds in baroclinic turbulence when both viscosity and a stable stratification are included.

Mathematical definitions.—We define the following statistical quantities. First, the helicity density is defined by $h=\boldsymbol{u} \cdot \boldsymbol{\omega}^{(a)}$ and the mean helicity by $\mathcal{H}_{1}=\langle h\rangle$. We recall that, in the framework of homogeneous turbulence, angular brackets are equivalent to space or ensemble averaging. Second, we define analogous quantities for the $\mathcal{B}$ vector. The scalar product between the $\mathcal{B}$ vector and the velocity field is denoted $\tilde{h}=\boldsymbol{u} \cdot \mathcal{B}$-called "crosshelicity" in [14]; it is the analog of the alignment between the velocity fluctuation and the magnetic field in MHDand we define in the same way the mean cross-helicity relative to $\mathcal{B}$ as $\tilde{\mathcal{H}}_{1}=\langle\tilde{h}\rangle$. In the particular case of homogeneous turbulence, the boundary surface integral in the equation for $d \tilde{\mathcal{H}}_{1} / d t$ vanishes, so that in the inviscid limit,

$$
\frac{d \tilde{\mathcal{H}}_{1}}{d t}=\left\langle\mathcal{B} \cdot b \boldsymbol{e}_{3}\right\rangle-\langle(2 \tilde{\Omega} \times \boldsymbol{u}) \cdot \mathcal{B}\rangle
$$

Lastly, we characterize the orientation between $\boldsymbol{u}, \boldsymbol{b} \boldsymbol{e}_{3}$, and $\mathcal{B}$ through the angles

$$
\begin{gathered}
\cos \theta=\|\boldsymbol{u}\|^{-1}\|\boldsymbol{\omega}\|^{-1} h, \\
\cos \tilde{\theta}=\|\boldsymbol{u}\|^{-1}\|\mathcal{B}\|^{-1} \tilde{h}, \\
\cos \tilde{\theta}_{\mathcal{P}}=|b|^{-1}\|\mathcal{B}\|^{-1} \mathcal{B} \cdot b \boldsymbol{e}_{3} .
\end{gathered}
$$

Numerical results.-Direct numerical simulations of homogeneous baroclinic turbulence are done on a $512 \times 768 \times 512$ grid. The numerical method is the Rogallo [20] extension of the Orszag-Patterson pseudospectral algorithm. Attention must be paid to the dealiasing

TABLE I. Numerical parameters. The final value of the Reynolds number based on the Taylor microscale $\lambda$ is denoted $R e_{\lambda}^{f}$. Turbulent kinetic energy is denoted $\mathcal{K}=\overline{u_{i} u_{i}} / 2$ and $\varepsilon$ is the dissipation. The ratio $S \mathcal{K} / \varepsilon$ therefore compares the linear shearing timescale to the nonlinear one. The Rossby number is defined by $R o=S / f$ and is close to 2 . From $N_{v}^{2} / S^{2}=0.8$ and up, baroclinic instability is triggered, leading to turbulent kinetic energy production [5].

\begin{tabular}{lcccccc}
\hline \hline Run & $R e_{\lambda}^{f}$ & $N_{v}^{2}$ & $N_{h}^{2}$ & $S \mathcal{K} / \varepsilon(0)$ & $S$ & $f$ \\
\hline A & 196 & 80 & 40 & 5.8 & 10 & 4 \\
B & 320 & 0.8 & 0.4 & 0.58 & 1 & 0.4 \\
C & 34 & 100 & 50 & 0.58 & 10 & 5 \\
\hline \hline
\end{tabular}

of potential vorticity when going back to spectral space. The classic $2 / 3$ rule is applied here. For details, the reader is referred to [21]. Numerical parameters are gathered in Table I. For all simulations A, B, and C, we use isotropic initial conditions coming from a decaying isotropic precalculation. During this phase, the buoyancy field is treated as a passive scalar. Thus, the isotropic buoyancy field at time $t S=0$ contains an initial amount of potential energy $\bar{b}^{2} / 2 \approx 6 \times 10^{-2}$. Then, the initial contribution of the fluctuating field to potential vorticity mainly reduces to its kinetic part-induced by the velocity field$\rho_{0} \Pi(0) \approx N^{2} \omega_{3}-S f \omega_{2}$. The initial kinetic energy content of symmetric modes represents about $7.2 \%$ of the total turbulent kinetic energy. We stress that the analogy and the results detailed in the present paper are quite general, and obtained for a widespread set of isotropic initial conditions for which the vortex mode is not initially zero (see [21] Sec. 4 for more details). For a discussion on the energetics of the instabilities in homogeneous baroclinic flows, the reader is referred to [5]. We first present the results obtained for cross-helicity or alignment between the velocity field $\boldsymbol{u}$ and the vector $\mathcal{B}$. The PDFs of $\cos \tilde{\theta}$ are presented in Fig. 2(a). The initial distribution is nonuniform due to the imposed background flow generating a strongly anisotropic potential vorticity gradient $\nabla \Pi$. Then, once baroclinic instability is triggered, the PDF evolves toward a more symmetric distribution but still with a clear preference for alignment $(\cos \tilde{\theta}=1)$. At $t S=20$, we find a $30 \%$
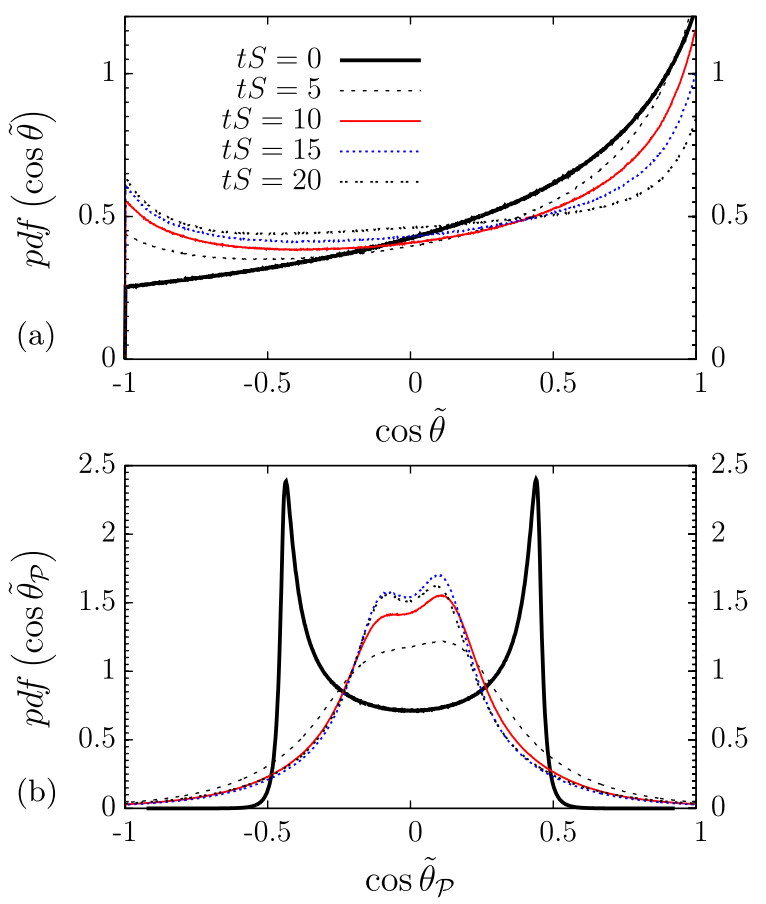

FIG. 2 (color online). Probability density function (PDF) of (a) $\cos \tilde{\theta}=\|\boldsymbol{u}\|^{-1}\|\mathcal{B}\|^{-1} \tilde{h}$, and (b) $\cos \tilde{\theta}_{\mathcal{P}}=|b|^{-1}\|\mathcal{B}\|^{-1} \mathcal{B} \cdot b \boldsymbol{e}_{3}$. Run $A$. 

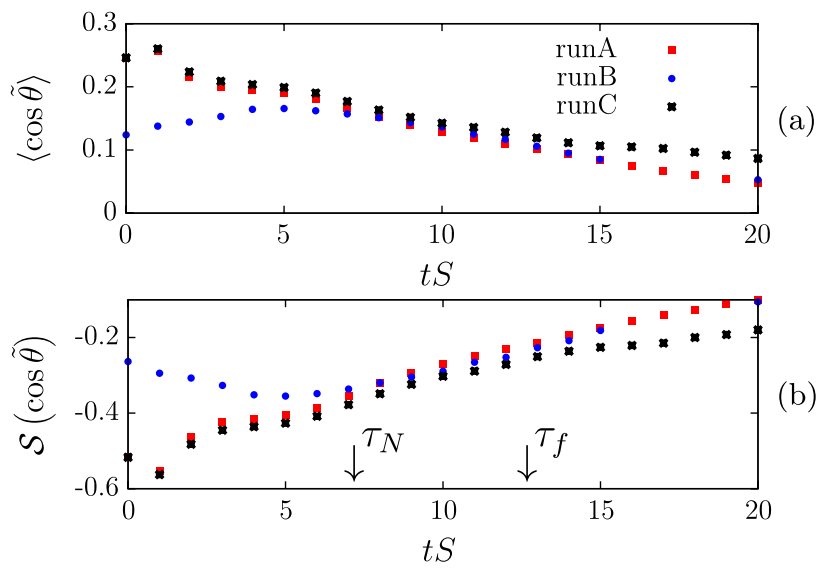

FIG. 3 (color online). Comparison between runs $A, B$, and $C$, showing the convergence toward zero of both (a) the mean $\langle\cos \tilde{\theta}\rangle$ and (b) the skewness $\mathcal{S}(\cos \tilde{\theta})$. Time $\tau_{N}$ corresponds to the characteristic stratification time $2 \pi / N_{v}$ where $N_{v}$ is the BruntVäisälä frequency in runs $A$ and $B$. In the same way, $\tau_{f}=2 \pi / f$ is the Coriolis characteristic time for run $C$.

difference between probabilities at $\cos \tilde{\theta}=-1$ and $\cos \tilde{\theta}=1$. For intermediate values of $\cos \tilde{\theta}$ —ranging from -0.5 to 0.5 - probability distribution is almost flat with an approximately $70 \%$ difference between probabilities at $\cos \tilde{\theta}=0$ and $\cos \tilde{\theta}=1$.

While mean normalized helicity $\langle\cos \theta\rangle$ is approximately zero-in our calculations $\mathcal{H}_{1} \simeq 10^{-2}$ for run $A$ and $\mathcal{H}_{1} \simeq$ $10^{-3}$ for run $B$-we get a significant amount of mean normalized cross-helicity. The results are presented in Fig. 3(a). For both runs $A$ and $B$ we observe a reduction of mean cross-helicity with time and the behavior after $t S=7$ is independent of $S \mathcal{K} / \varepsilon(0)$. This time scale thus appears to be of the order of the stratification time scale $\tau_{N} S=2 S \pi / N_{v} \approx 7$. The same is true for the skewness factor as highlighted by Fig. 3(b). The skewness $\mathcal{S}(\cos \tilde{\theta})$ converges to zero by negative values meaning that the PDF is skewed in favor of positive values of $\cos \tilde{\theta}$ but becomes more symmetric when baroclinic turbulence develops. Looking at run $C$, the same behavior is observed but with a slower convergence rate after the Coriolis time $\tau_{f} S \approx 12.5$.

The same analysis is applied to $\cos \tilde{\theta}_{\mathcal{P}}$, the angle between $\mathcal{B}$ and the gravity axis. We show in Fig. 2(b) that the distribution of $\cos \tilde{\theta}_{\mathcal{P}}$ when turbulence has developed after $t S=20$ is quite symmetric but favors dramatically perpendicular orientations of $\mathcal{B}$ with the gravity axis, i.e., values such that $\cos \tilde{\theta}_{\mathcal{P}} \approx 0$.

Equation (8) suggests that we investigate the joint probability distribution of $\cos \tilde{\theta}_{\mathcal{P}}$ and $\cos \tilde{\theta}$. The results are presented in Fig. 4. For both runs $A$ and $B$, it is shown that orthogonality of $\mathcal{B}$ and the gravity axis is associated to negative (positive) cross-helicity. Net preference for positive cross-helicity is confirmed: light red regions are located in the upper half of the graph. It is also clear that the highest values of $\cos \tilde{\theta}$ are gathered on the right side of the PDF, i.e., when $\cos \tilde{\theta}_{\mathcal{P}}$ is positive. We also found (not shown) that the $\mathcal{B}$ vector also tends to be orthogonal to the Coriolis force. Besides, the contribution of the Coriolis term to cross-helicity production dominates the dynamics. A picture of the vorticity field and cross-helicity distribution is given in Fig. 5. Vortical regions in Fig. 5(a) are tilted and located around the mean isobuoyancy surfaces. In Fig. 5(b) the dominant red color attests to a preferential alignment between $\boldsymbol{u}$ and $\mathcal{B}$. It also shows that this alignment takes place at small scales.

Conclusion.-We have studied developed homogeneous baroclinic turbulence with high-resolution DNS. Alignment between the vector $\mathcal{B}=\nabla \Pi \times \nabla(\bar{b}+b)$ and the velocity field, called cross-helicity, is shown to be nonzero on average. In particular, a preference for alignment between the velocity field $\boldsymbol{u}$ and the $\mathcal{B}$ vector is highlighted. Based on the work by [14], we propose a new scenario that explains this feature whereby positive crosshelicity is generated in regions where the $\mathcal{B}$ vector is aligned with the gravity axis, so that the angle $\tilde{\theta}_{\mathcal{P}}$ plays a crucial role for cross-helicity dynamics, as also stressed by

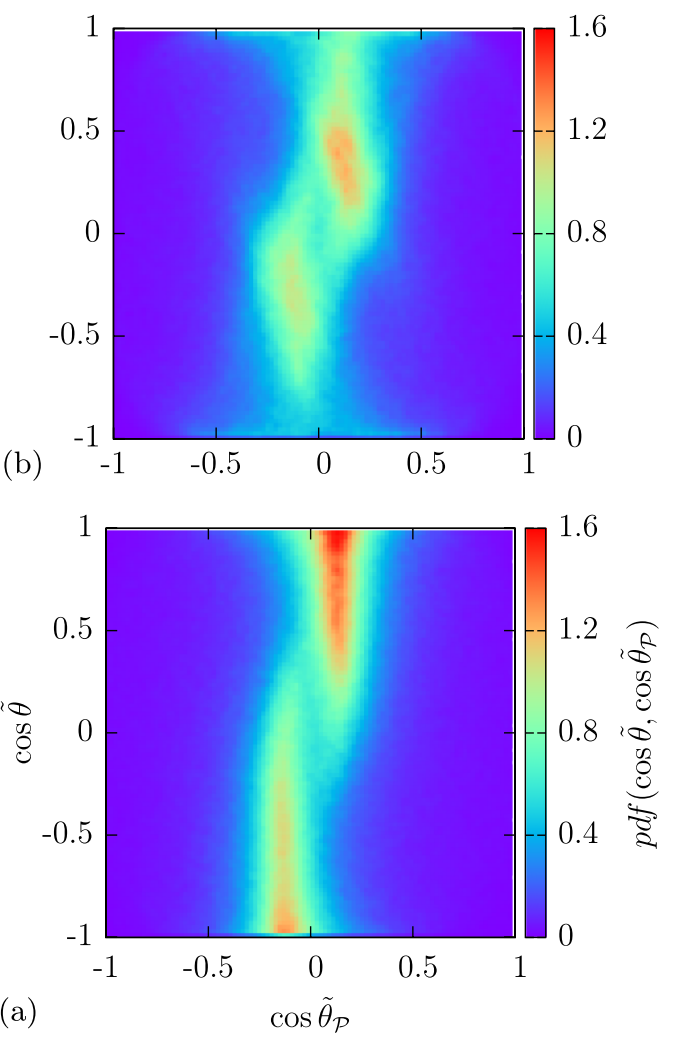

FIG. 4 (color online). Joint probability distributions of $\cos \tilde{\theta}$ and $\cos \tilde{\theta}_{\mathcal{P}}$ for (a) run $A$ and (b) run $B$ at time $t S=20$. The figures show that orthogonality between $\mathcal{B}$ and $b \boldsymbol{e}_{3}$ is linked to antialignment of $\mathcal{B}$ with the velocity field $\boldsymbol{u}$ independently of the shear intensity $S$. 


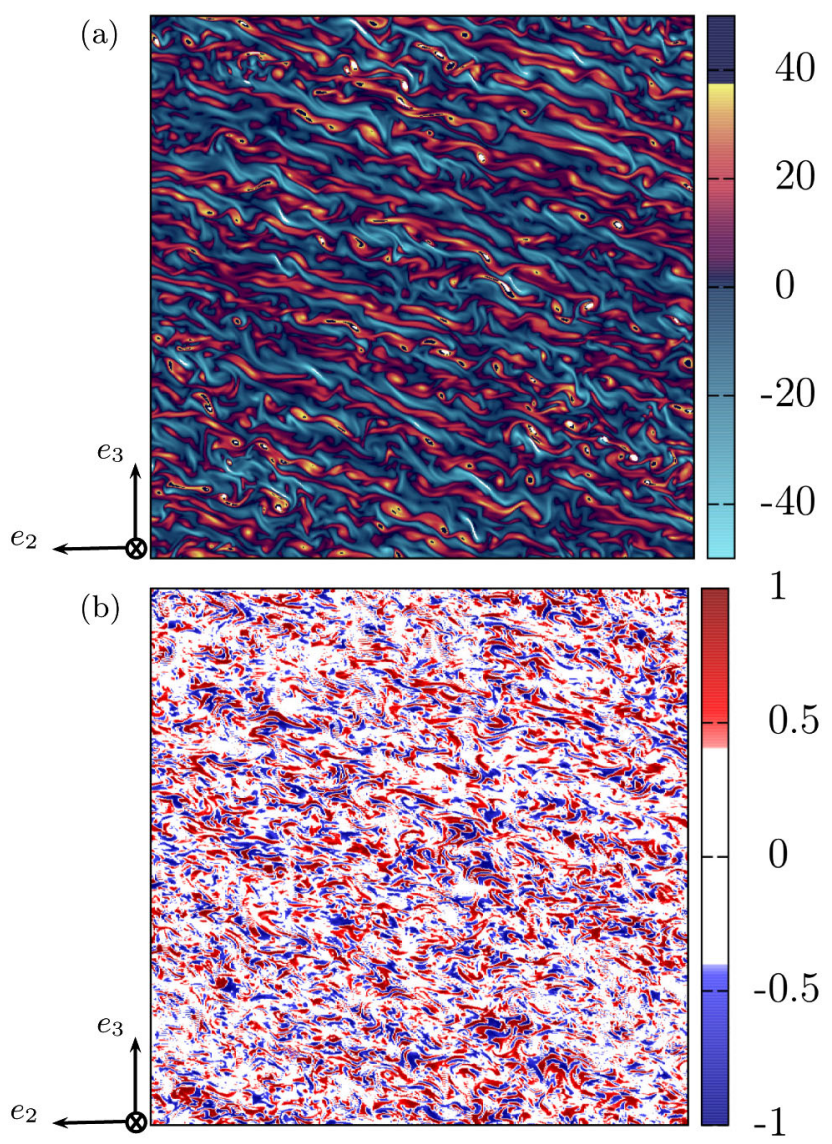

FIG. 5 (color online). (a) Zonal vorticity in the vertical or meridional plane at time $t S=20$, run $A$. (b) The corresponding distribution of $\cos \theta$ defined in Eq. (10). The slope of the vortical structures is approximately $24.5^{\circ}$, which is close to the baroclinic angle $\operatorname{atan}\left(N_{h}^{2} / N_{v}^{2}\right)=26.6^{\circ}$.

Eq. (8). Besides, in the first stage of turbulence, the evolution of first- and third-order moments of $\cos \tilde{\theta}$ are sensitive to the intensity of the mean background gradients (Fig. 3). However, after the time $t S=\tau_{N}$ it is shown that cross-helicity's PDFs tend to symmetrize and equilibrate alignment and antialignment independently of the background gradients (for both runs $A$ and $B$ ).

This tendency toward uniformization of the PDF of $\cos \tilde{\theta}$ is not due to the baroclinic instability mechanism, as shown in Fig. 3, since the stable case $\mathrm{C}$ follows the same tendency.

Finally, the dynamics of the potential vorticity gradient is essential in geophysical fluid dynamics. The links with cross-helicity dynamics and statistical mechanics developed in this Letter open new perspectives for a better understanding of rotating stratified flows. In particular, our new results on cross-helicity dynamics, here derived in the idealized case of a homogeneous baroclinic flow, can be extended to more complex geophysical contexts.

The DNS results have been obtained at IDRIScomputing center of the French National Research Council (CNRS)-under Project No. 26683. A. B. P. acknowledges financial support by the Institute of Atmospheric Sciences and Climate (ISAC, Italy) under Project No. ISAC-005-2012-TO.

*a.pieri@isac.cnr.it

[1] J. Pedlosky, Geophysical Fluid Dynamics (Springer-Verlag, Berlin, 1982).

[2] P. G. Drazin and W. H. Reid, Hydrodynamic stability (Cambridge University Press, Cambridge, England, 2004).

[3] A. Salhi and C. Cambon, J. Appl. Mech. 73, 449 (2006).

[4] A. B. Pieri, C. Cambon, F. S. Godeferd, and A. Salhi, Phys. Fluids 24, 076603 (2012).

[5] A. B. Pieri, F. S. Godeferd, C. Cambon, and A. Salhi, J. Fluid Mech. 734, 535 (2013).

[6] R. T. Pierrehumbert and K. L. Swanson, Annu. Rev. Fluid Mech. 27, 419 (1995).

[7] H. K. Moffatt, J. Fluid Mech. 35, 117 (1969).

[8] U. Frisch, Turbulence: The Legacy of A. N. Kolmogorov (Cambridge University Press, Cambridge, England, 1996).

[9] R. B. Pelz, V. Yakhot, S. A. Orszag, L. Shtilman, and E. Levich, Phys. Rev. Lett. 54, 2505 (1985).

[10] A. Tsinober, E. Kit, and T. Dracos, Advances in Turbulence 3, 514 (1991).

[11] J. M. Wallace and J. L. Balint, "An Experimental Study of Helicity and Related Properties in Turbulent Flows," in IUTAM Symposium on Topological Fluid Mechanics (Cambridge University Press, Cambridge, England, 1989), Vol. 1.

[12] R. Monchaux, F. Ravelet, B. Dubrulle, A. Chiffaudel, and F. Daviaud, Phys. Rev. Lett. 96, 124502 (2006).

[13] J. C. Perez and S. Boldyrev, Phys. Rev. Lett. 102, 25003 (2009).

[14] J. D. Gibbon and D. D. Holm, J. Phys. A 43, 172001 (2010).

[15] E. Heifetz and B. F. Farrell, J. Atmos. Sci. 60, 2083 (2003).

[16] N. A. Bakas and P. J. Ioannou, J. Atmos. Sci. 64, 1509 (2007).

[17] G. K. Vallis, Atmospheric and Oceanic Fluid Dynamics: Fundamentals and Large-Scale Circulation (Cambridge University Press, Cambridge, England, 2006).

[18] S. Thalabard, Ph.D. thesis, CEA Saclay, 2013.

[19] P. H. Chavanis and J. Sommeria, J. Fluid Mech. 356, 259 (1998).

[20] R. S. Rogallo, Numerical Experiments in Homogeneous Turbulence (National Aeronautics and Space Administration, 1981), Vol. 81315.

[21] A. B. Pieri, Ph.D. thesis, Ecole Centrale de Lyon, Université de Lyon, 2012. 